\title{
Association of Cord Blood Magnesium Concentration and Neonatal Resuscitation
}

\author{
Lynn H. Johnson, M. D., Delicia C. Mapp, M.S., Dwight J. Rouse, M.D., Catherine Y. Spong, \\ M.D., Brian M. Mercer, M.D., Kenneth J. Leveno, M.D., Michael W. Varner, M.D., Jay D. lams, \\ M.D., Yoram Sorokin, M.D., Susan M. Ramin, M.D., Menachem Miodovnik, M.D., Mary J. \\ O'Sullivan, M.D., Alan M. Peaceman, M.D., Steve N. Caritis, M.D., and the Eunice Kennedy \\ Shriver National Institute of Child Health and Human Development Maternal-Fetal Medicine \\ Units Network ${ }^{*}$ \\ Department of Pediatrics, University of North Carolina at Chapel Hill, Chapel Hill, NC; from the \\ Department of Obstetrics and Gynecology at the University of Alabama at Birmingham, \\ Birmingham, AL; Case Western Reserve University-MetroHealth Medical Center, Cleveland, OH; \\ University of Texas Southwestern Medical Center, Dallas, TX; University of Utah, Salt Lake City, \\ Utah: The Ohio State University, Columbus, $\mathrm{OH}$; Wayne State University, Detroit, MI; University \\ of Texas Health Science Center at Houston, Houston, TX; University of Cincinnati, Cincinnati, \\ $\mathrm{OH}$; University of Miami, Miami, FL; Northwestern University, Chicago, IL; University of \\ Pittsburgh, Pittsburgh, PA; and The George Washington University Biostatistics Center, \\ Washington, DC; and the Eunice Kennedy Shriver National Institute of Child Health and Human \\ Development, Bethesda, MD
}

\begin{abstract}
Objective Assess the relationship between umbilical cord blood magnesium concentration and level of delivery room resuscitation received by neonates.

Study design Secondary analysis of a controlled fetal neuroprotection trial that enrolled women at imminent risk for delivery between 24 and 31 weeks' gestation and randomly allocated them to receive intravenous magnesium sulfate or placebo. The cohort included 1507 infants for whom total cord blood magnesium concentration and delivery room resuscitation information were available. Multivariable logistic regression was used to estimate the association between cord blood magnesium concentration and highest level of delivery room resuscitation, using the following hierarchy: none, oxygen only, bag-mask ventilation with oxygen, intubation or chest compressions.
\end{abstract}

\footnotetext{
(C) 2011 Mosby, Inc. All rights reserved.

Address for Correspondence: Lynn H. Johnson, M.D., Division of Neonatal-Perinatal Medicine, UNC Hospitals, CB\#7596, Chapel Hill, NC 27599-7596, lynn.johnson@me.com..

*A list of members of the Eunice Kennedy Shriver National Institute of Child Health and Human Development Maternal-Fetal Medicine Units Network is available at www.jpeds.com (Appendix).

Publisher's Disclaimer: This is a PDF file of an unedited manuscript that has been accepted for publication. As a service to our customers we are providing this early version of the manuscript. The manuscript will undergo copyediting, typesetting, and review of the resulting proof before it is published in its final citable form. Please note that during the production process errors may be discovered which could affect the content, and all legal disclaimers that apply to the journal pertain.

The authors declare no conflicts of interest.
} 
Results-There was no relationship between cord blood magnesium and delivery room resuscitation (odds ratio [OR] 0.92 for each $1.0 \mathrm{mEq} / \mathrm{L}$ increase in magnesium; $95 \%$ confidence interval [CI]: 0.83-1.03). Maternal general anesthesia was associated with increased neonatal resuscitation (OR 2.51; 95\% CI: 1.72-3.68). Each 1-week increase in gestational age at birth was associated with decreased neonatal resuscitation (OR 0.63; 95\% CI: $0.60-0.66$ ).

Conclusion-Cord blood magnesium concentration does not correlate with the level of delivery room resuscitation of infants exposed to magnesium sulfate for fetal neuroprotection.

\section{Keywords}

magnesium; cord blood; delivery room resuscitation

Magnesium is implicated in numerous physiologic and pharmacologic roles including the reduction of myometrial and other smooth muscle contractility, depression of CNS irritability, stabilization of cellular membranes and interference with acetylcholine release at the neuromuscular junction.(1-3) For decades, pregnant women have received magnesium sulfate (MgSO4) for either tocolysis or the prevention of eclampsia. Recently, multiple studies have demonstrated that antenatal treatment with $\mathrm{MgSO} 4$ reduces the risk of cerebral palsy among children born preterm.(4-6)

Adverse effects may occur in women who become hypermagnesemic during $\mathrm{MgSO} 4$ treatment. These include muscular weakness, diminished deep tendon reflexes and respiratory depression.(2) Similar adverse effects may occur in neonates exposed to $\mathrm{MgSO} 4$ antenatally. In clinical practice, infants whose mothers received $\mathrm{MgSO} 4$ for any indication are thought to be at risk for respiratory depression, and thus an increased need for delivery room resuscitation. For example, case reports suggest a relationship between fetal $\mathrm{MgSO} 4$ exposure and the need for resuscitation immediately after birth. $(7,8)$ To date, however, hypothesized adverse effects on neonates have not been proven. Despite the lack of definitive evidence linking fetal magnesium exposure and the need for resuscitation, the American Academy of Pediatrics and American Heart Association supported Neonatal Resuscitation Program (NRP) lists MgSO4 among maternally administered medications that might depress respirations in the newborn.(9)

Our objective in this secondary analysis of the largest trial of magnesium sulfate for fetal neuroprotection was to estimate the relationship between umbilical cord blood magnesium concentration and the need for resuscitation of infants immediately after delivery. In the primary analysis of the trial, there was no overall association between $\mathrm{MgSO} 4$ exposure and increased delivery room resuscitation.(4) However, this finding does not preclude a concentration-dependent effect. Therefore, in this investigation, we evaluated the association between cord blood magnesium concentration and delivery room resuscitation.

\section{METHODS}

This was a secondary analysis of the Eunice Kennedy Shriver National Institute of Child Health and Human Development Maternal Fetal Medicine Units (MFMU) Network trial of MgSO4 for the prevention of cerebral palsy.(4) From December 1997 through May 2004, 
women at imminent risk for preterm delivery between 24 and 31 weeks' gestation were randomized to receive intravenous $\mathrm{MgSO} 4$ or placebo. Magnesium sulfate (or matching placebo) was given to study participants intravenously as a 6 gram loading dose over 20 minutes followed by 2 grams infused each hour in minimal volume intravenous fluids. The infusion could be increased to a maximum of 4 grams per hour at the discretion of the physician and was continued for at least 12 hours if delivery did not occur. Retreatment with the study drug was given for recurrence of labor or anticipated delivery unless criteria for exclusion developed in the interim. The primary outcome was the composite of stillbirth or death by 1 year corrected age or moderate or severe cerebral palsy at 2 years corrected age. Details of the study are reported elsewhere.(4) Mother/neonate dyads included in the current analysis were enrolled in the original trial and had both cord blood magnesium concentration and delivery room resuscitation data recorded. Neonates who fulfilled these criteria were included in this analysis irrespective of the mother's allocation to magnesium or placebo. The institutional review board at each of the 20 participating centers approved participation in the trial. All participants gave written informed consent before enrollment.

Trained, certified research nurses collected data prospectively and included information on both maternal and neonatal characteristics, complications and outcomes. Data describing other medications given during labor, including anesthetic agents and analgesics, were abstracted from the mothers' charts. Gestational age at entry to the trial was determined according to a standard method predicated on the date of the last menstrual period and the results of the earliest available ultrasound.(10) Umbilical cord blood was collected immediately after delivery and serum was separated within 45 to 120 minutes following collection. Samples were frozen within 12 hours from the time the blood was collected and stored locally at $-70^{\circ} \mathrm{C}$ until shipping to a central laboratory (Quest Diagnostics, Van Nuys, California). Total magnesium was measured using the Olympus chemistry analyzer, valid low $=0.4 \mathrm{mEq} / \mathrm{L}(0.2 \mathrm{mmol} / \mathrm{L})$.

Neonatal delivery data recorded included the highest level of delivery room resuscitation received by the infant. The hierarchy of this variable (from lowest to highest) was none, followed by oxygen only, bag-mask ventilation with oxygen, intubation or chest compressions. All infants, including those labeled with a resuscitation variable of "none," received standard delivery room care.(9)

\section{Statistical analyses}

Statistical analyses were performed using SAS® statistical software (SAS institute, Cary, NC). The primary outcome was the highest level of delivery room resuscitation received by the infant. The primary exposure was cord blood magnesium concentration. Generalized estimating equations were used to account for the correlations between siblings of twin pregnancies. Proportional odds models within this framework allowed for regression analyses to measure the association between magnesium and the five-level ordinal resuscitation variable (none, oxygen only, bag-mask ventilation, intubation or chest compressions). These regression models were used for univariate analyses, as well as for multivariable analyses that controlled for gestational age, administration of maternal narcotics and general anesthesia. Odds ratios (OR; 95\% Confidence Interval) were 
calculated for each of the predictor variables. Similar models were constructed with the dichotomous outcome of none vs. any resuscitation using ordinary logistic regression.

\section{RESULTS}

In the parent study, 2444 fetuses were randomized at 20 sites between December 1997 and May 2004. Of these, 924 were excluded from this secondary analysis because cord blood was unavailable either because clinical staff were unable to obtain it or there was an insufficient amount of blood available after other testing. There were 13 stillbirths. Our study cohort comprised 1507 infants with both cord blood magnesium concentration and delivery room resuscitation data. A total of 723 mothers (48.0\%) were assigned to $\mathrm{MgSO} 4$ and 784 mothers (52.0\%) were assigned to placebo. Neonates in the present analysis were of more advanced gestational age, of higher birth weight and more often delivered vaginally than neonates for whom cord blood magnesium concentration was not available (Table I). The mean gestational age at delivery was $30.0 \pm 2.9$ weeks; only $28(1.9 \%)$ infants were delivered after 37 weeks.

Of the 1507 infants, 252 (16.7\%) received no additional resuscitation (Table II). For infants who received any form of resuscitation, oxygen only and intubation were the most common; $432(28.7 \%)$ and 528 (35.0\%) infants, respectively. Relatively few infants required chest compressions, $\mathrm{n}=46(3.1 \%)$. Decreasing birth weight and gestational age at birth were associated with an increasing receipt of resuscitation. Infants who received chest compressions had a mean birth weight of $1050 \mathrm{~g}$, whereas those who received oxygen and those who did not receive any additional resuscitation had mean birth weights of $1660 \mathrm{~g}$ and $1883 \mathrm{~g}$, respectively. The mean gestational age at birth decreased with increasing level of resuscitation $(\mathrm{p}<0.001)$. Infants who received intubation or chest compressions tended to have lower 1- and 5-minute Apgar scores and greater exposure to general anesthesia.

Table III represents the univariate analysis of the highest level of resuscitation in relation to cord blood magnesium concentration. The mean magnesium concentration for the cohort was $2.05 \pm 0.88 \mathrm{mEq} / \mathrm{L}$ which did not significantly differ across the highest level of resuscitation recorded $(\mathrm{p}=0.28)$. Of the 1507 infants, $51(3.4 \%)$ had magnesium concentrations greater than $4.0 \mathrm{mEq} / \mathrm{L}$; only $6(0.4 \%)$ had concentrations greater than 5.0 $\mathrm{mEq} / \mathrm{L}$. Additional (any) delivery room resuscitation was received by $45(88.2 \%)$ of these 51 infants. The resuscitation received by the 6 infants with a magnesium concentration of $>5.0$ $\mathrm{mEq} / \mathrm{L}$ included intubation (4), bag/mask (1) or oxygen (1).

We included cord blood magnesium concentration, gestational age at birth, administration of maternal narcotics, and general anesthesia in a multivariable logistic regression analysis to determine the association between the highest level of resuscitation (using the 5-level ordinal model) and cord blood magnesium concentration (Table IV). Cord blood magnesium concentration was not associated with an increased need for delivery room resuscitation (OR 0.92 for each $1.0 \mathrm{mEq} / \mathrm{L}$ increase in magnesium; 95\% CI: 0.83-1.03). Maternal general anesthesia was associated with increased delivery room resuscitation (OR 2.51; 95\% CI: 1.72-3.68) but maternal administration of narcotics was not (OR 0.97; 95\% CI: 0.80-1.19). 
For each 1-week increase in gestational age at birth there was decreased receipt of delivery room resuscitation (OR 0.63; 95\% CI: $0.60-0.66$ ).

An additional regression analysis was performed with resuscitation as a dichotomous variable, none vs. any, to again look for an association between the highest level of resuscitation and cord blood magnesium concentration. This model similarly included gestational age at birth, cord blood magnesium concentration, general anesthesia and maternal narcotics. This model yielded identical findings to the 5-level ordinal model, with no association between cord blood magnesium concentration and receipt of delivery room resuscitation (data not shown).

\section{DISCUSSION}

In the setting of $\mathrm{MgSO} 4$ administration for neuroprotection in anticipated preterm birth, we found no association between umbilical cord blood magnesium concentration and the receipt of an increased level of delivery room resuscitation in newborn infants, after adjusting for gestational age at birth and several intrapartum exposures. Exposure to general anesthesia was associated with a greater degree of neonatal resuscitation, an association that has previously been reported.(11-13) Not surprisingly, increasing gestational age at birth was associated with decreased receipt of delivery room resuscitation, consistent with previous reports.(13-15)

Previous studies appear to support our observation that cord blood concentrations of magnesium do not predict the need for neonatal resuscitation. For example, Lipsitz and English reported on 16 neonates with effects of hypermagnesemia, including mild respiratory depression, hypotonia and hyporeflexia following maternal exposure to $\mathrm{MgSO} 4$ for preeclampsia.(16) However, they found no correlation between Apgar scores or other clinical signs and cord blood magnesium concentration or need for resuscitation. In a case controlled study, Riaz et al reported a non-statistically significant increase in delivery room support, defined as bag-mask ventilation, in $26 \mathrm{MgSO} 4$ exposed infants.(17) They reported these infants more likely to be hypotonic and to have lower Apgar scores. However, there was no association of these outcomes with cord blood magnesium concentration, maternal magnesium concentration, or total dose or duration of maternal MgSO4 exposure. In the parent trial for this secondary analysis, there was no statistically significant difference in rates of generalized hypotonia or the composite outcome of intubation and chest compressions among magnesium vs. placebo exposed infants.(4) Along with our results, these studies suggest that fetal $\mathrm{MgSO} 4$ exposure does not have adverse effects on delivery room resuscitation. Rather, the confounding circumstances that are associated with maternal $\mathrm{MgSO} 4$ treatment (e.g., early gestational age) may increase the likelihood of respiratory depression immediately after birth, thus leading to increased receipt of delivery room resuscitation.

Published literature related to intrapartum magnesium sulfate exposure has primarily addressed the neonate born to mothers with preeclampsia or eclampsia as the indication for magnesium exposure.(17-19) Few studies have focused exclusively on the preterm infant, and most have reported only Apgar scores or clinically subjective assessment scales as 
surrogate markers of the presumed impact of magnesium exposure on delivery room resuscitation. Despite reports of the effects of magnesium exposure on tone, Apgars or clinical assessment, none of these studies found a correlation between cord blood or serum magnesium concentration and the degree of neonatal depression.

There are several strengths and limitations to our study. We included a large number of infants. The magnesium concentration was measured at birth, and thus is the most accurate reflection of fetal magnesium levels. Clinicians who were resuscitating the infant were unaware of treatment assignment, and thus bias was limited. As for limitations, infants in our study had relatively low levels of magnesium in the cord blood (mean $2.05 \pm 0.88$ $\mathrm{mEq} / \mathrm{L})$, compared with other studies with a range $1.8-11.5 \mathrm{mg} / \mathrm{dL}(1.5 \mathrm{mEq} / \mathrm{L}-9.6 \mathrm{mEq} / \mathrm{L})$. $(16-18,20)$ Thus it is possible that very high concentrations of cord blood magnesium might be associated with a greater need for resuscitation. Of the 1507 infants, $51(3.4 \%)$ had magnesium concentrations greater than $4.0 \mathrm{mEq} / \mathrm{L}$ and only $6(0.4 \%)$ of these had concentrations greater than $5.0 \mathrm{mEq} / \mathrm{L}$, with the majority of these infants receiving additional delivery room resuscitation. Thus, our analysis is not powered to look for an association at higher concentrations. It is worth emphasizing, however, that antenatal magnesium administration in the dose range utilized for neuroprotection rarely resulted in high cord blood magnesium concentrations, so even if a subsequent association were found, it would be applicable to only a small minority of infants exposed to magnesium sulfate for neuroprotection.

We did not investigate other reported side effects of MgSO4 exposure, such as apnea/ hypoventilation following admission or delayed feeding tolerance or ileus. In addition, our findings might not be applicable to term infants because our cohort was primarily composed of preterm infants, with only 28 (1.9\%) infants greater than 37 weeks gestation at delivery. The threshold for delivery room intubation of extremely preterm infants (i.e. for surfactant administration) might vary across sites, however these data were not collected as part of this study.

In conclusion, we found no association between umbilical cord blood magnesium concentration and receipt of delivery room resuscitation of infants. This finding should motivate clinicians to further investigate an etiology for greater level of delivery room resuscitation, and to consider other potential contributing causes for the need for resuscitation. The findings of this analysis should allow providers to provide magnesium sulfate for neuroprotection without the concern that it will lead to additional invasive delivery room resuscitation measures.

\section{Acknowledgments}

The following subcommittee members participated in protocol development and coordination between clinical research centers (Allison T. Northen, M.S.N., R.N.), protocol/data management and statistical analysis (Elizabeth Thom, Ph.D. and Steven J. Weiner, M.S.), and protocol development and oversight (Deborah G. Hirtz, M.D. and John M. Thorp, Jr., M.D.). Matthew M. Laughon, M.D., M.P.H. (supported by the US government for his work in pediatric and neonatal clinical pharmacology [NIH grant 1K23HL092225-01]), University of North Carolina at Chapel Hill, provided additional support with analysis and review of the manuscript.

Supported by grants from the Eunice Kennedy Shriver National Institute of Child Health and Human Development (NICHD) (HD27869, HD34208, HD34116, HD40544, HD27915, HD34136, HD21414, HD27917, HD27860, 
HD40560, HD40545, HD40485, HD40500, HD27905, HD27861, HD34122, HD40512, HD53907, HD34210, HD21410, HD36801, HD19897]MO1-RR-000080) and the National Institute of Neurological Disorders and Stroke (NINDS). This report does not necessarily represent the official views of the NICHD, NINDS or the National Institutes of Health.

\section{Appendix}

In addition to the authors, other members of the Eunice Kennedy Shriver National Institute of Child Health and Human Development Maternal-Fetal Medicine Units Network are as follows:

University of North Carolina at Chapel Hill, Chapel Hill, NC - J. Thorp, K.J. Moise, Jr., S. Brody, J. Bernhardt, K. Dorman

University of Alabama at Birmingham, Birmingham, AL - J.C. Hauth, A. Northen, T. HillWebb, S. Tate, K. Nelson, F. Biasini

University of Utah, Salt Lake City, UT - L. Fullmer, K. Anderson, A. Guzman, M. Jensen, L. Williams

University of Texas Southwestern Medical Center, Dallas, TX - M.L. Sherman, J. Dax, L. Faye-Randall, C. Melton, E. Flores

Case Western Reserve University-MetroHealth Medical Center, Cleveland, OH - M. Collin, G. VanBuren, C. Milluzzi, M. Fundzak, C. Santori

The Ohio State University, Columbus, $\mathrm{OH}$ - F. Johnson, M.B. Landon, C. Latimer, V. Curry, S. Meadows

Thomas Jefferson University, Philadelphia, PA - R.J. Wapner, A. Sciscione, M.M. DiVito, M. Talucci, S. Desai, D. Paul

University of Tennessee, Memphis, TN - B.M. Sibai, R. Ramsey, W. Mabie, L. Kao, M. Cassie

Wayne State University, Detroit, MI - G.S. Norman, D. Driscoll, B. Steffy, M.P.

Dombrowski

Wake Forest University Health Sciences, Winston-Salem, NC - P.J. Meis, M. Swain, K. Klinepeter, M. O'Shea, L. Steele

University of Texas Health Science Center at Houston, Houston, TX - L.C. Gilstrap, III, M.C Day, E. Gildersleve, F. Ortiz, M. Kerr

Columbia University, New York, NY - F. Malone, V. Pemberton, L. Paley, C. Paley, S. Bousleiman, V. Carmona

Brown University, Providence, RI - M. Carpenter, J. Tillinghast, D. Allard, B. Vohr, L. Noel, K. McCarten

University of Cincinnati, Cincinnati, $\mathrm{OH}$ - N. Elder, W. Girdler, T. Gratton 
University of Chicago, Chicago, IL - A.H. Moawad, M. Lindheimer, P. Jones

University of Miami, Miami, FL - F. Doyle, C. Alfonso, M. Scott, R. Washington

Northwestern University, Chicago, IL - G. Mallett, M. Ramos-Brinson., P. Simon

University of Texas Medical Branch, Galveston, TX - G. Hankins, L.A. Goodrum, G.R. Saade, G.L. Olson, H.M. Harirah, E. Martin

University of Texas at San Antonio, San Antonio, TX - O. Langer, E. Xenakis, D. Conway, M. Berkus

University of Pittsburgh, Pittsburgh, PA - T. Kamon, M. Cotroneo, C. Milford

The George Washington University Biostatistics Center - E. Thom, S.J. Weiner, B. JonesBinns, M. Cooney, M. Fischer, S. McLaughlin, K. Brunette, E. Fricks

National Institute of Neurological Disorders and Stroke, Bethesda, MD - D.G. Hirtz, K.B. Nelson

Eunice Kennedy Shriver National Institute of Child Health and Human Development, Bethesda, MD - S. Tolivaisa, D. McNellis, C. Catz, K. Howell

MFMU Network Steering Committee Chair (University of Pittsburgh, Pittsburgh, PA) - J. Roberts

\section{REFERENCES}

1. Mercer BM, Merlino AA. Magnesium sulfate for preterm labor and preterm birth. Obstet Gynecol. 2009; 114:650-68. [PubMed: 19701047]

2. Ramsey PS, Rouse DJ. Magnesium sulfate as a tocolytic agent. Semin Perinatol. 2001; 25:236-47. [PubMed: 11561911]

3. Hirtz DG, Nelson K. Magnesium sulfate and cerebral palsy in premature infants. Curr Opin Pediatr. 1998; 10:131-7. [PubMed: 9608889]

4. Rouse DJ, Hirtz DG, Thom E, Varner MW, Spong CY, Mercer BM, et al. A randomized, controlled trial of magnesium sulfate for the prevention of cerebral palsy. N Engl J Med. 2008; 359:895-905. [PubMed: 18753646]

5. Crowther CA, Hiller JE, Doyle LW, Haslam RR. Effect of magnesium sulfate given for neuroprotection before preterm birth: a randomized controlled trial. JAMA. 2003; 290:2669-76. [PubMed: 14645308]

6. Marret S, Marpeau L, Zupan-Simunek V, Eurin D, Leveque C, Hellot MF, et al. Magnesium sulphate $\mathrm{P}$ given before very-preterm birth to protect infant brain: the randomised controlled PREMAG trial*. BJOG. 2007; 114:310-8. [PubMed: 17169012]

7. Teng RJ, Liu HC, Tsou Yau KI. Neonatal hypermagnesemia: report of one case [abstract]. Zhonghua Min Guo Xiao Er Ke Yi Xue Hui Za Zhi. 1989; 30:333-6. [PubMed: 2637615]

8. Herschel M, Mittendorf R. Tocolytic magnesium sulfate toxicity and unexpected neonatal death. J Perinatol. 2001; 21:261-2. [PubMed: 11533846]

9. Kattwinkel, J.; Boyle, D.; Bloom, RS. 5th ed.. American Academy of Pediatrics; Elk Grove Village, Ill.: 2006. American Heart Association., American Academy of Pediatrics. Textbook of neonatal resuscitation..

10. Carey JC, Klebanoff MA, Hauth JC, Hillier SL, Thom EA, Ernest JM, et al. Metronidazole to prevent preterm delivery in pregnant women with asymptomatic bacterial vaginosis. National 
Institute of Child Health and Human Development Network of Maternal-Fetal Medicine Units. N Engl J Med. 2000; 342:534-40. [PubMed: 10684911]

11. Mattingly JE, D'Alessio J, Ramanathan J. Effects of obstetric analgesics and anesthetics on the neonate : a review. Paediatr Drugs. 2003; 5:615-27. [PubMed: 12956618]

12. Levy BT, Dawson JD, Toth PP, Bowdler N. Predictors of neonatal resuscitation, low Apgar scores, and umbilical artery pH among growth-restricted neonates. Obstet Gynecol. 1998; 91:909-16. [PubMed: 9610995]

13. Niermeyer S, Kattwinkel J, Van Reempts P, Nadkarni V, Phillips B, Zideman D, et al. International Guidelines for Neonatal Resuscitation: An excerpt from the Guidelines 2000 for Cardiopulmonary Resuscitation and Emergency Cardiovascular Care: International Consensus on Science. Contributors and Reviewers for the Neonatal Resuscitation Guidelines. Pediatrics. 2000; 106:E29. [PubMed: 10969113]

14. MacDonald HM, Mulligan JC, Allen AC, Taylor PM. Neonatal asphyxia. I. Relationship of obstetric and neonatal complications to neonatal mortality in 38,405 consecutive deliveries. $\mathrm{J}$ Pediatr. 1980; 96:898-902. [PubMed: 7365599]

15. Aziz K, Chadwick M, Baker M, Andrews W. Ante- and intra-partum factors that predict increased need for neonatal resuscitation. Resuscitation. 2008; 79:444-52. [PubMed: 18952348]

16. Lipsitz PJ, English IC. Hypermagnesemia in the newborn infant. Pediatrics. 1967; 40:856-62. [PubMed: 6075658]

17. Riaz M, Porat R, Brodsky NL, Hurt H. The effects of maternal magnesium sulfate treatment on D newborns: a prospective controlled study. J Perinatol. 1998; 18:449-54. [PubMed: 9848759]

18. Rasch DK, Huber PA, Richardson CJ, L'Hommedieu CS, Nelson TE, Reddi R. Neurobehavioral effects of neonatal hypermagnesemia. J Pediatr. 1982; 100:272-6. [PubMed: 7199083]

19. Rantonen T, Ekblad U, Gronlund J, Rikalainen H, Valimaki I, Kero P. Influence of maternal magnesium E sulphate and ritodrine treatment on the neonate: a study with six-month follow-up. Acta Paediatr. 1999; 88:1142-6. [PubMed: 10565464]

20. Lipsitz PJ. The clinical and biochemical effects of excess magnesium in the newborn. Pediatrics. 1971; 47:501-9. [PubMed: 5547870] 
Table 1

Neonatal Characteristics and Intrapartum Care: Comparison of Those With and Without Available Cord Blood Magnesium Concentration

\begin{tabular}{|c|c|c|c|}
\hline & Mg Concentration Not Available $\mathbf{n = 9 2 4}$ & Mg Concentration Available $n=1507$ & P-Value \\
\hline Gestational age, wk ${ }^{*}$ & $29.3 \pm 3.4$ & $30.0 \pm 2.9$ & $<0.001$ \\
\hline Birth weight, g & $1324 \pm 615$ & $1472 \pm 539$ & $<0.001$ \\
\hline Birth weight, $\leq 1500 \mathrm{~g}$ & $633(68.7)$ & $828(54.9)$ & $<0.001$ \\
\hline Female & $437(47.3)$ & $710(47.1)$ & 0.93 \\
\hline \multicolumn{4}{|l|}{ Mode of Delivery } \\
\hline Vaginal (1456) & $502(54.4)$ & $954(63.3)$ & $<0.001$ \\
\hline Cesarean (973) & $420(45.6)$ & $553(36.7)$ & \\
\hline \multicolumn{4}{|l|}{ Race } \\
\hline African-American & $400(43.3)$ & $645(42.8)$ & 0.74 \\
\hline Caucasian & $330(35.7)$ & $580(38.5)$ & \\
\hline Hispanic & $176(19.0)$ & $245(16.3)$ & \\
\hline Asian & $4(0.4)$ & $16(1.1)$ & \\
\hline Native American & $1(0.1)$ & $2(0.1)$ & \\
\hline Other & $13(1.4)$ & $9(1.3)$ & \\
\hline 1-minute Apgar & $6(4-8)$ & $7(5-8)$ & $<0.001$ \\
\hline 1-minute Apgar $<5$ & 293 (31.9) & $302(20.1)$ & $<0.001$ \\
\hline 5-minute Apgar & $8(7-9)$ & $8(7-9)$ & 0.09 \\
\hline 5-minute Apgar $<7$ & $225(24.5)$ & $229(15.2)$ & $<0.001$ \\
\hline General Anesthesia & $101(12.5)$ & $103(7.3)$ & $<0.001$ \\
\hline Maternal Narcotics & $297(36.7)$ & $521(37.1)$ & 0.90 \\
\hline Twin Gestation & $91(11.1)$ & $112(8.0)$ & $<0.001$ \\
\hline
\end{tabular}

Data are presented as mean $\pm \mathrm{SD}, \mathrm{n}(\%)$ or median (interquartile range)

* Gestational age at delivery 


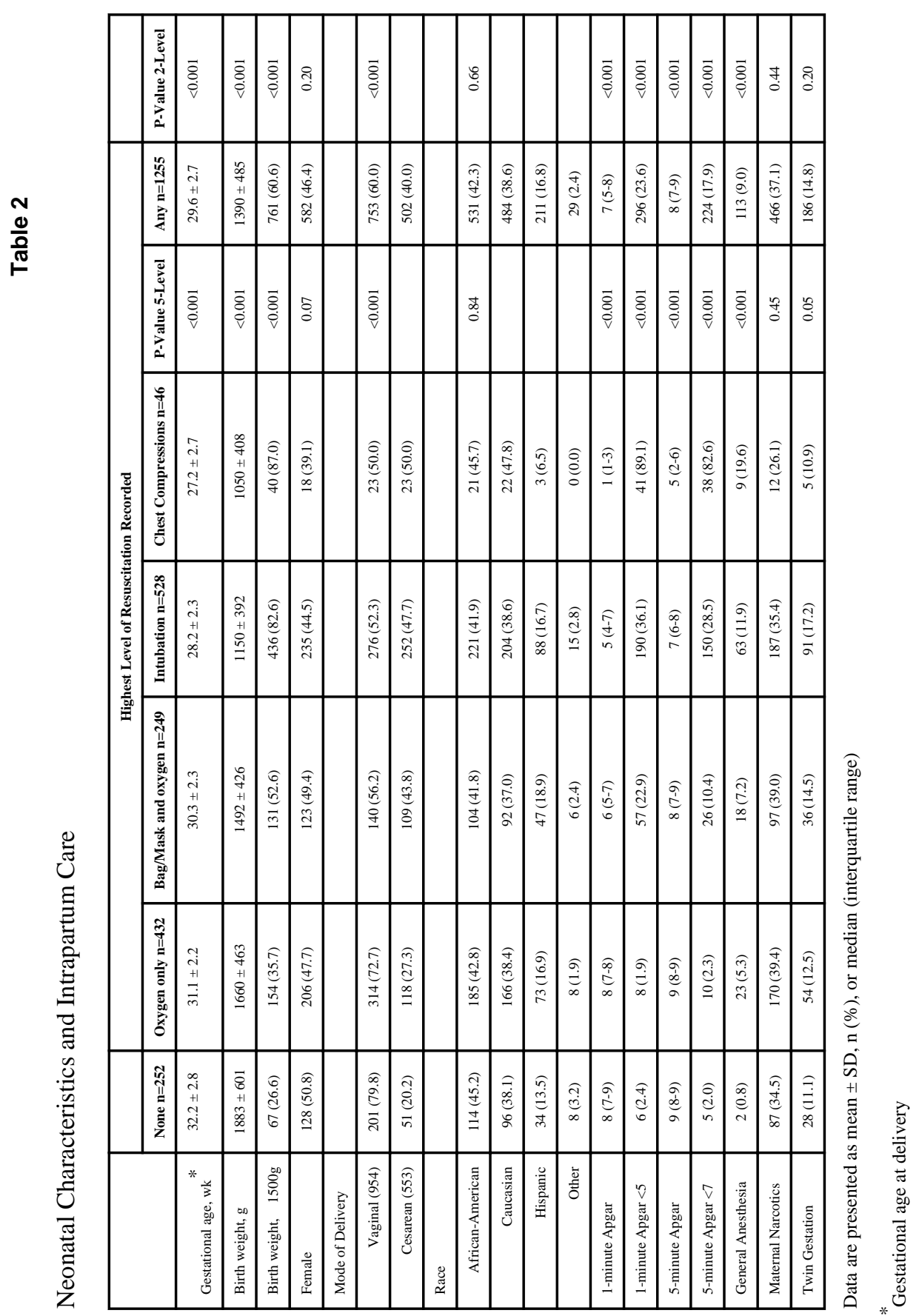


Table 3

Level of Resuscitation by Cord Blood Magnesium Concentration

\begin{tabular}{|c|c|c|c|c|c|c|}
\hline \multirow{2}{*}{ Magnesium concentration (mEq/L) } & \multicolumn{5}{|c|}{ Highest Level of Resuscitation Recorded } \\
\cline { 2 - 7 } & None $\mathbf{n = 2 5 2}$ & Oxygen only $\mathbf{n = 4 3 2}$ & $\begin{array}{c}\text { Bag/ } \\
\text { Mask } \\
\text { and } \\
\text { oxygen } \\
\mathbf{n}=\mathbf{2 4 9}\end{array}$ & Intubation n=528 & Chest Compressions n=46 & Any $\mathbf{n = 1 2 5 5}$ \\
\hline$<1.0, \mathrm{n}=52$ & $12(23.1)$ & $13(25.0)$ & $10(19.2)$ & $15(28.9)$ & $2(3.9)$ & $40(76.9)$ \\
\hline $1.0-<2.0, \mathrm{n}=900$ & $167(18.6)$ & $257(28.6)$ & $134(14.9)$ & $317(35.2)$ & $25(2.8)$ & $733(81.4)$ \\
\hline $2.0-<3.0, \mathrm{n}=279$ & $38(13.6)$ & $66(23.7)$ & $57(20.4)$ & $108(38.7)$ & $10(3.6)$ & $241(86.4)$ \\
\hline $3.0-<4.0, \mathrm{n}=225$ & $29(12.9)$ & $75(33.3)$ & $42(18.7)$ & $70(31.1)$ & $9(4.0)$ & $196(87.1)$ \\
\hline $4.0-<5.0, \mathrm{n}=45$ & $6(13.3)$ & $20(44.4)$ & $5(11.1)$ & $14(31.1)$ & $3(0)$ & $39(86.7)$ \\
\hline $25.0, \mathrm{n}=6$ & $0(0)$ & $1(16.7)$ & $1(16.7)$ & $4(66.7)$ & $6(0)$ & $6(100.0)$ \\
\hline Mean Magnesium concentration & $1.9 \pm 0.81$ & $2.1 \pm 0.96$ & $2.1 \pm 0.88$ & $2.0 \pm 0.86$ & $2.1 \pm 0.81$ & $2.1 \pm 0.89$ \\
\hline
\end{tabular}

Data are presented as number $(\%)$ or mean \pm standard deviation

Mean magnesium concentration did not differ across the highest level of resuscitation recorded, $\mathrm{p}=0.28$ 


\section{Table 4}

Multivariable Analysis of Risk for Delivery Room Resuscitation ${ }^{*}$

\begin{tabular}{|l|l|l|}
\hline Variable & Odds Ratio & 95\% Confidence Interval \\
\hline Magnesium $^{* *}$ & 0.92 & $0.83-1.03$ \\
\hline General Anesthesia & 2.51 & $1.72-3.68$ \\
\hline Maternal Narcotics & 0.97 & $0.80-1.19$ \\
\hline Gestational Age $^{* * *}$ & 0.63 & $0.60-0.66$ \\
\hline
\end{tabular}

* Analysis of delivery room resuscitation as a 5-level ordinal variable and adjusted for magnesium, general anesthesia, maternal narcotics and gestational age

** For each $1.0 \mathrm{mEq} / \mathrm{L}$ interval increase in cord magnesium concentration

$* * *$

For each 1-week increase in gestational age 